\title{
Deux applications de l'analyse factorielle à des données géotechniques
}

\section{P. POUGET}

N. CADOUX*

Laboratoire régional des ponts et chaussées 8-10, rue Bernard-Palissy BP 11 63014 Clermont-Ferrand * Stagiaire CUST $-3^{\circ} \mathrm{A}$

J.-P. MAGNAN

Laboratoire central des ponts et chaussées 58 , bd Lefebvre 75732 Paris Cedex 15
La méthode d'analyse factorielle a été appliquée à l'analyse des données géotechniques provenant de deux sites : une zone de sols argileux compressibles où un grand nombre d'essais œdométriques a été réalisé dans un sondage et une zone de sols surconsolidés et peu déformables dans laquelle se trouve une vallée fossile remplie de sédiments argileux mous. Le premier site a été analysé pour trouver les relations existant entre les paramètres et définir les frontières des couches. Le second site a été analysé pour retrouver la géométrie de la vallée fossile à partir de mesures au pénétromètre statique. Ces deux exemples illustrent les possibilités de I'analyse factorielle, qui a fourní le résultat cherché dans les deux cas. Ils ont aussi mis en évidence quelques sources d'erreurs possibles.

Mots clés : analyse statistique, analyse factorielle, site compressible, vallée fossile, exemples.

\section{Two applications of factor analysis to geotechnical data}

The factor analysis method has been applied to the analysis of geotechnical data from two sites : a zone of compressible clavey soils where a large number of oedemeter compressibility tests were made and a zone of overconsolidated stiff soils where a hidden valley filled up with soft clayey sediments was found. The first site was analysed with the intention to find relationships between the soil pararneters and to define the layer boundaries. The second site was analysed in order to find the geometry of the hidden valley from come penetration tests. The two examples show the possibilities offered by factor analysis, which gave the intended results. They displayed possible sources of errors, too.

Key words : statistical analysis, factor analysis, compressible site, hidden valley, examples. 


\section{Introduction}

Malgré les nombreux travaux qui leur ont été consacrés, les méthodes d'analyse de données restent pour les géotechniciens des outils mal connus et utilisés de façon très marginale, sous forme notamment de calculs statistiques élémentaires tels que les calculs de moyenne et de variance et les études de régression linéaire, où la statistique porte sur la variabilité des données autour d'une droite définie par la méthode des moindres carrés.

L'analyse factorielle, utilisée dès les années 1970 pour des applications de géotechnique (Favre, 1980), offre des possibilités complémentaires pour déterminer les relations existant à l'intérieur d'un ensemble de paramètres. Les deux études présentées dans cet article ont été réalisées sur cleux sites où de très nombreuses données étaient disponibles :

- la première a été effectuée sur le site expérimental de Cubzac-les-Ponts (sols argileux compressibles), déjà analysé par Baghery et Magnan (1983) par des méthodes d'analyse de corrélations et de régression linéaire. Il s'agissait de voir si l'utilisation de l'analyse factorielle, sous la forme de l'analyse en composantes principales, apportait des résultats différents des analyses déjà effectuées :

- la seconde étude portait sur la recherche automatique d'ensembles de terrains homogènes dans la reconnaissance géotechnique d'un site. Cette seconde application a été réalisée sur les données d'une étude géotechnique exécutée quelques années plus tôt et qui avait mis en évidence l'existence d'une vallée fossile remplie de sols compressibles dans un environnement de sols surconsolidés et résistants.

Ces deux applications sont décrites successivement dans cet article, après un rappel succinct des principes de l'analyse factorielle de données.

\section{2}

\section{La méthode d'analyse factorielle (rappels)}

L'analyse factorielle cherche à représenter la variabilité d'un ensemble de n variables aléatoires (X) par un plus petit nombre p d'autres variables $\left[Y_{i}\right]_{-=1}$ appelées catégories ou facteurs. Ce travail se fait à partir de $m$ observations des variables $X_{r}$, soit $\left(x_{i k}\right\}_{i=1, n, k=1}$

Pour atteindre cet objectif, on construit par étapes une suite de variables $Y$, qui représentent chacune l'essentiel de la variabilité restante des données. Ce travail peut être représenté comme la recherche des axes principaux des $m$ points observés dans l'espace à $n$ dimensions d'axes $\left(X_{1}, X_{2}, \ldots X\right)$. Pour $n=3$, cette procédure est représentée sur la figure 1 :

- la première phase est la recherche de l'axe $Y_{,}$, qui passe par le centre de gravité $G$ des points et minimise la somme des carrés des distances des points $\left(x_{i j}\right)$ à ce premier axe; - la deuxième phase est la recherche de l'axe principal d'inertie $\mathrm{Y}_{2}$ dans le plan passant par $\mathrm{G}$ et perpendiculaire à l'axe $\mathrm{Y}_{1}$. La procédure est la même que pour $\mathrm{Y}_{1}$ : on cherche l'orientation de l'axe $Y_{2}$ qui minimise la somme des carrés des distances des points au plan $\mathrm{Y}_{1} \mathrm{Y}_{2}$;
- le troisième axe est perpendiculaire aux deux premiers.

Quand il existe plus de trois variables X, la procédure est la mème avec un axe principal $Y_{\text {f }}$ et des axes successifs définis par minimisation par rapport à des hyperplans.

L'expression mathématique de la procédure de recherche des axes (Y) est décrite dans divers ouvrages (Bertier et Bouroche, 1977 ; Dagnelie, 1977 ; Lebart et al., 1982). Elle est brièvement rappelée ci-après.

La relation cherchée entre les $n$ variables $(X)$ et les $p$ variables ( $Y$ ) peut s'écrire sous la forme générale :

$$
\begin{aligned}
& \left(\begin{array}{c}
Y_{1} \\
\ldots \\
Y_{p}
\end{array}\right)=\left(\begin{array}{ccc}
u_{11} & \ldots & u_{1 n} \\
\ldots & u_{j 1} & \ldots \\
u_{p 1} & \ldots & u_{p n}
\end{array}\right)\left(\begin{array}{c}
X_{1} \\
\ldots . \\
X_{n}
\end{array}\right)+\left(\begin{array}{c}
\varepsilon_{1} \\
\ldots \\
\varepsilon_{p}
\end{array}\right) \\
& |Y\rangle=\quad|U| \quad|X|+|\varepsilon|
\end{aligned}
$$

oủ $\{\varepsilon]$ est la matrice des résidus (écarts entre les valeurs réelles et les valeurs calculées) de la relation. La matrice [U], pour sa part, est la matrice de passage du repère orthonormé des variables $|\mathrm{X}|$ au repère orthonormé des nouvelles variables $[\mathrm{Y}]$. Cette matrice contient les coordonnées en ligne des vecteurs unitaires des axes (Y) par rapport à la base des vecteurs unitaires des axes [X]. Compte tenu de son origine, la matrice (U) a les propriétés suivantes :

$$
\sum_{i} u_{j i}^{2}=1
$$

(vecteurs unitaires)

et

$\sum \mathrm{u}_{\mathrm{j}} \mathrm{u}_{\mathrm{ki}}=0$ pour $\mathrm{j} \# \mathrm{k}$ (vecteurs orthogonaux par deux).

Par conséquent la matrice (U) est telle que $\langle U|\langle U\rangle^{\prime}=\langle 1|$ (matrice unité) et $|U|^{\prime}$ est l'inverse de |U|.

Les points $P_{k}$ observés ( $m$ valeurs des $\left(X_{i} \mid\right.$, soit $\left(x_{j}\right)$ ) ont pour coordonnées dans le nouveau repère des $(Y)$ :

$\left.\mid \mathrm{y}_{j \mathrm{k}}\right)=\left(\mathrm{u}_{\mu}\right)\left[\mathrm{x}_{\mathrm{ik}}\right)$.

Dans ces matrices, les coordonnées $\left\{x_{i w} \mid\right.$ des points $\mathrm{P}_{\mathrm{k}}$ sont rangées en colonnes et les $\left(\mathrm{y}_{i k}\right)$ correspondants en lignes.

Si l'origine des axes est placée au centre de gravité $G$ des points $P_{x^{\prime}}$ la distance entre le point $G$ et la protection $\mathrm{H}_{\mathrm{k}}$ d'un point $\mathrm{P}_{1}$ sur l'axe portant la variable $\mathrm{Y}_{\text {, }}$ (Fig. 2) vaut :

$$
\left|y_{i k}\right|=\left|u_{i p}\right|\left\lfloor x_{i k}\right]
$$

Le carré de cette distance est égal à :

$$
\begin{aligned}
& \left|\mathrm{GH}_{k+1}\right|^{2}=\left\{\mathrm{u}_{11}\right\}\left\{\mathrm{x}_{i k}\right\}\left\{\mathrm{x}_{i \mathrm{k}}\right\}^{1}\left\{\mathrm{u}_{11}\right\}^{1} \\
& =\sum \mathrm{u}_{11}^{2} \mathrm{x}_{i k}^{2}
\end{aligned}
$$

La somme $\Sigma^{2}$ des carrés des distances $\mathrm{GH}_{\mathrm{k}, 1}$ pour tous les points $\mathrm{P}_{\mathrm{k}}$ est donnée par le produit de matrices

$$
\Sigma^{2}=\left[y_{i k}\right)\left(y_{i k}\right)^{t}=\left(u_{i j}\right)\left(x_{i k}\right)\left(x_{i k}\right)^{t}\left(u_{i j}\right)^{t} \text {. }
$$



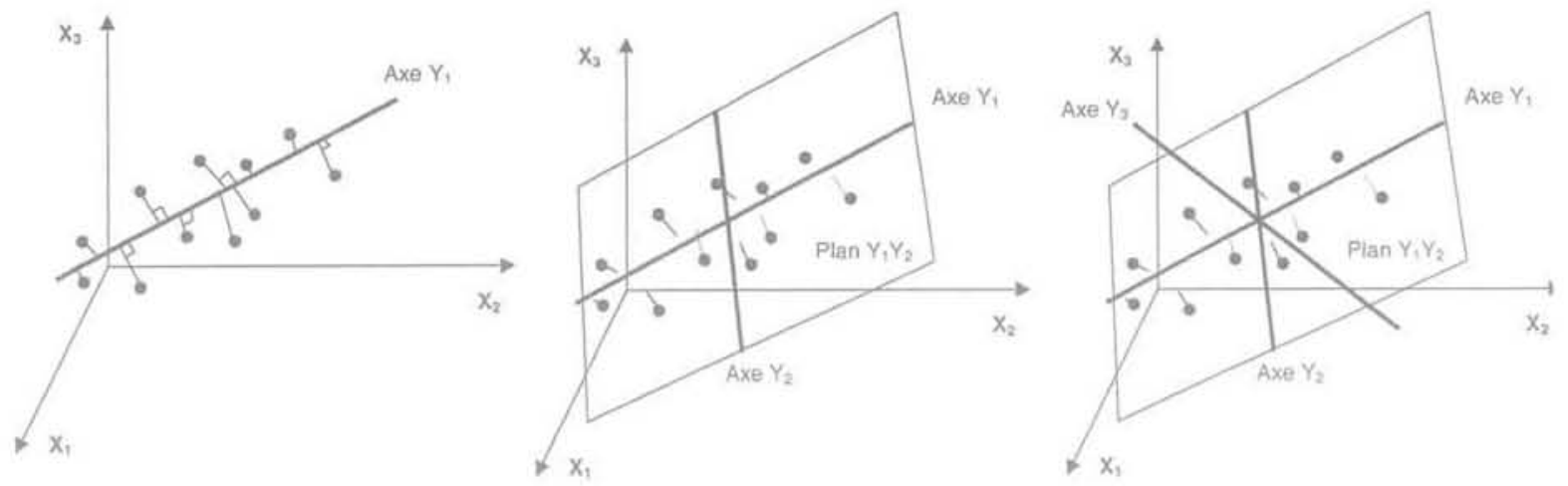

Fig. Procédure de recherche des axes principaux d'inertie. Procedure for finding the principal inertia axes.

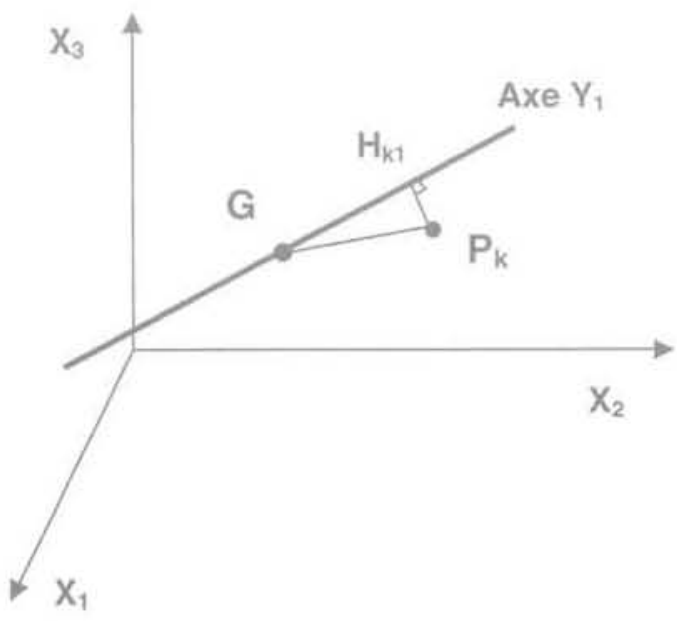

Fig. 2 Distance du point $\mathrm{P}_{\mathrm{k}}$ à l'axe $\mathrm{Y}_{4}$. Distance from point $P_{k}^{*}$ to axis $Y_{1}$.

La somme des carrés des distances des points $\mathrm{P}_{\mathrm{k}}$ à l'axe GY, est égale à :

$$
\sum_{k} H_{k 1} P_{k}^{2}=\sum_{k} G P_{k}^{2}-\sum_{k} G H_{j a}^{2} .
$$

Comme $\sum_{k} \mathrm{GP}_{k}^{2}$ est constant pour un ensemble de points $\mathrm{P}_{\mathrm{k}}$ donné, minimiser $\sum_{k} \mathrm{H}_{\mathrm{k} 1} \mathrm{P}_{\mathrm{k}}^{2}$ revient à maximiser $\sum_{k} \mathrm{GH}_{\mathrm{k} 1}{ }^{2}=\Sigma^{2}$.

Pour maximiser $\Sigma^{2}$ par le choix optimal de l'axe GY, on procède de la façon suivante.

On recherche le vecteur unitaire de GY, de composantes $\left\{\mathrm{u}_{1}\right\}$ sur la base orthonormée des $[\mathrm{X}\}$ qui maximise le produit $\left(\mathrm{u}_{\mathrm{i}}\right)\left(\mathrm{x}_{i \mathrm{k}}\right)\left(\mathrm{x}_{i \mathrm{k}}\right)^{\mathrm{t}}\left(\mathrm{u}_{\mathrm{i}}\right)^{\mathrm{t}}$.

La matrice $(A)=\left(x_{i}\right)\left(x_{k}\right)^{\prime}$ est symétrique et définie positive, donc diagonalisable, avec des valeurs propres positives. Soit (B) la matrice des coordonnées en colonnes des vecteurs propres unitaires de $|A|$ dans le repère des $\left(\mathrm{X}_{i}\right)$ et $(\Lambda)$ la matrice de ses valeurs propres:

$$
[\mathrm{B}]=\left(\mathrm{b}_{w}\right) \quad\{\Lambda\}=\left[\begin{array}{ccc}
\lambda_{1} & 0 & 0 \\
0 & \lambda_{\alpha} & 0 \\
0 & 0 & \lambda_{n}
\end{array}\right] \text {. }
$$

On peut écrire : d'où :

$[\mathrm{A})(\mathrm{B})=(\mathrm{B})(\mathrm{A})$

$(A)=(B)(A)(B)^{t}$ car $(B)(B)^{t}=(1)$.

Si l'on écrit les coordonnées des vecteurs propres unitaires $\left\{b_{\alpha i}\right\}$ dans leur repère (qui est celui des $Y_{\text {, cher- }}$ chés), on obtient une nouvelle matrice:

$$
[C]=\left(C_{a j}\right)=(B)^{t}(U)^{t} \text {, }
$$

et l'on peut écrire que

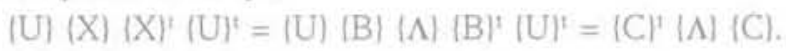
La condition $(\mathrm{U})^{t}(\mathrm{U}\rangle=\{1\rangle$ devient alors

$\langle C)^{*}(C)=\left.(\mathrm{U})(\mathrm{B})^{(\mathrm{B})}\right|^{\mathrm{t}}(\mathrm{U})^{\mathrm{t}}=(\mathrm{U})(\mathrm{U})^{\mathrm{t}}=(1)$.

La maximisation de $(\mathrm{U})(\mathrm{X})(\mathrm{X})^{\prime}(\mathrm{U})^{\mathrm{t}}$ pour la première direction propre correspond donc à celle de l'expression :

$$
\sum_{\alpha} \lambda_{\alpha} c_{\alpha 1}^{2}
$$

sous la condition $\sum_{a} c_{\alpha]}^{2}=1$.

En effet, les $\mathrm{c}_{\text {a }}$ sont les coordonnées des vecteurs propres unitaires sur la première direction propre. On peut remplir les conditions imposées en choisissant la direction propre associée à la plus grande valeur propre $\lambda$. On a ainsi déterminé l'orientation du premier facteur $Y_{1}$.

La procédure se continue en utilisant successivement les valeurs propres et directions propres dans l'ordre décroissant des valeurs propres.

Si l'on détermine également les valeurs propres $\mu_{\alpha}$ de la matrice $X^{\prime} X$ et les vecteurs propres unitaires $v_{a}$. correspondants, on dèmontre que :

$$
\begin{gathered}
\lambda_{\alpha}=\mu_{\alpha} \\
X v_{\alpha}=\sqrt{\lambda_{\alpha}} u_{\alpha},
\end{gathered}
$$

d’où

$$
\mathrm{X}=\sum_{\alpha=1}^{\alpha=p} \sqrt{\lambda_{\alpha} v_{\alpha} \mathrm{u}_{\alpha}^{\dagger}} .
$$

Le taux d'inertie ou part de variance expliquée

$$
\tau_{p}=\sum_{\alpha=1}^{p} \lambda_{\alpha} / \sum_{\alpha=1}^{n} \lambda_{\alpha}
$$


permet d'apprécier la qualité de la représentation des $\mathrm{X}_{1}$ par les $\mathrm{Y}_{\mathrm{i}}$.

\section{3}

\section{Applications, généralités}

Les analyses en composantes principales présentées dans cet article ont été réalisées au moyen du logiciel STATGRAPHICS. Le calcul fournit:

- un vecteur contenant les valeurs propres, qui expriment la variance de chaque composante principale;

- une matrice contenant les vecteurs propres, qui sont les pondérations à appliquer aux variables pour obtenir les facteurs;

- une matrice contenant les corrélations entre les variables de départ et les facteurs (utile à l'interprétation des facteurs).

La projection des individus et des caractères est réalisée sur l'espace principal considéré. En général, comme nous avons pu le vérifier, le plan défini par les deux premiers axes factoriels comprend un pourcentage important de l'information.

L'interprétation des résultats et de leurs représentations graphiques conduit aux informations suivantes : - l'origine des axes $\mathrm{G}$ est le centre de gravité du nuage: il représente la tendance moyenne des individus. Les individus situés loin du centre présentent un écart important par rapport à cette moyenne et influencent beaucoup les statistiques ;

- l'inertie d'un axe, représentée par la valeur propre associée à son vecteur directeur, témoigne de la représentativité de cet axe par rapport au nuage des points: plus l'inertie du plan est forte, plus la projection du nuage sur ce plan est réaliste avec un minimum de perte d'information:

- les coordonnées des points sur les axes factoriels sont les coefficients de corrélation des individus qu'ils représentent avec ces axes. La valeur du produit scalaire des vecteurs joignant deux points à l'origine est le coefficient de corrélation entre les deux variables correspondantes: des vecteurs voisins indiquent des variables fortement corrélées ;

- la proximité, dans le plan, d'individus et de variables n'a pas de signification. Un lien entre ces paramètres nécessite une interprétation de la signification des facteurs.

\section{4}

\section{Analyse de la compressibilité de l'argile molle de Cubzac}

Lors de l'étude géotechnique des sols du site expérimental des Laboratoires des Ponts et Chaussées à Cubzac-les-Ponts, un sondage carotté de $20 \mathrm{~cm}$ de diamètre et $9 \mathrm{~m}$ de longueur a été entièrement consacré à l'étude des variations des résultats des essais œdométriques en fonction de la profondeur (Sondage A44, Remblai D, Haghgou, 1983) : on dispose ainsi de 168 résultats d'essais œedométriques, à raison de deux essais au même niveau tous les $6,5 \mathrm{~cm}$ de profondeur, avec des interruptions périodiques dues au carottage (remaniement des extrémités des carottes).
L'étude générale du site a montré que la coupe des sols dans la zone du sondage A44 est la suivante :

- de 0 à 1,3 m, couche superficielle d'argile molle surconsolidée, correspondant aux fluctuations annuelles de la nappe phréatique ;

- de 1,3 à 2,5 m, vase limono-argileuse moyennement organique, très compressible et légèrement surconsolidée :

- de 2,5 à $9 \mathrm{~m}$, vase argilo-limoneuse faiblement organique, très compressible et légèrement surconsolidée, plus dense de 2,5 à $5,2 \mathrm{~m}\left(\mathrm{w}=65 \%, \gamma=16 \mathrm{kN} / \mathrm{m}^{3}\right)$ que de 5,2 à $9 \mathrm{~m}\left(\mathrm{w}=80 \%, \gamma=14,8 \mathrm{kN} / \mathrm{m}^{3}\right)$.

Les données géotechniques du site expérimental de Cubzac-les-Ponts ont été analysées par Baghery et Magnan (1983) et par Haghgou (1983), en utilisant les méthodes classiques de la statistique et notamment l'analyse de régression linéaire. L'étude présentée ici cherchait à savoir si l'analyse factorielle pouvait apporter des informations complémentaires par rapport à l'analyse de régression linéaire.

Le travail d'analyse en composantes principales a été effectué sur un tableau comportant 168 lignes et 13 colonnes :

- colonne 1 : i, numéro de l'éprouvette ;

- colonne 2 : $\mathrm{z}$, profondeur $(\mathrm{m})$;

- colonne 3 : w, teneur en eau naturelle $(\%)$;

- colonne $4: \gamma$, poids volumique $\left(\mathrm{kN} / \mathrm{m}^{3}\right)$;

- colonne $5: \gamma_{\mathrm{d}}$, poids volumique du sol $\sec \left(\mathrm{kN} / \mathrm{m}^{3}\right)$;

- colonne 6: $e_{\sigma^{\prime}}$ indice des vides en place ;

- colonne $7: \mathrm{C}_{s}$, indice de gonflement;

- colonne 8: $\mathrm{C}_{\mathrm{c}}$, indice de compression ;

- colonne 9: $\sigma^{\prime}$ pression de préconsolidation $(\mathrm{kPa})$; - colonne 10: $c_{v}$, coefficient de consolidation verticale $\mathrm{m}^{2} / \mathrm{s}$ )

- colonne $11: \mathrm{k}_{v}$, coefficient de perméabilité verticale $(\mathrm{m} / \mathrm{s})$;

- colonne 12: $e_{k}$, paramètre de la relation entre e et $k_{\gamma}$ $\left(\mathrm{e}=\mathrm{e}_{\mathrm{k}}+\mathrm{C}_{\mathrm{k}} \lg \mathrm{k}_{\mathrm{v}}\right)$;

- colonne 13: $C_{k}$, paramètre de la relation entre e et $k_{v}$.

L'analyse en composantes principales réalisée sur l'ensemble des données a montré que les deux premiers axes factoriels représentaient toujours de 70 à $100 \%$ de l'information initiale. L'exposé des résultats sera pour cette raison limité à ces deux axes.

\section{1}

\section{Analyse au niveau des paramètres}

L'analyse réalisée sur l'ensemble des mesures entre $1,085 \mathrm{~m}$ et $9,075 \mathrm{~m}$ de profondeur montre un plan factoriel (Fig, 3) dont l'inertie totale est de 72,6\% (59,5\% pour le premier axe, $13,1 \%$ pour le deuxième). Sur ce plan sont représentés simultanément les points correspondant aux individus et les vecteurs correspondant aux variables. Quatre groupes de vecteurs rapprochés les uns des autres peuvent être observés. Ils sont associés respectivement aux variables suivantes :

$$
\begin{aligned}
& -\gamma \text { et } \gamma_{d^{\prime}} \\
& -w, e_{\sigma^{\prime}} C_{c^{\prime}} e_{k} \text { et } C_{k^{\prime}} \\
& -c_{v} \text { et } k_{v^{\prime}} \\
& -z \text { et } \sigma_{p^{\prime}}
\end{aligned}
$$




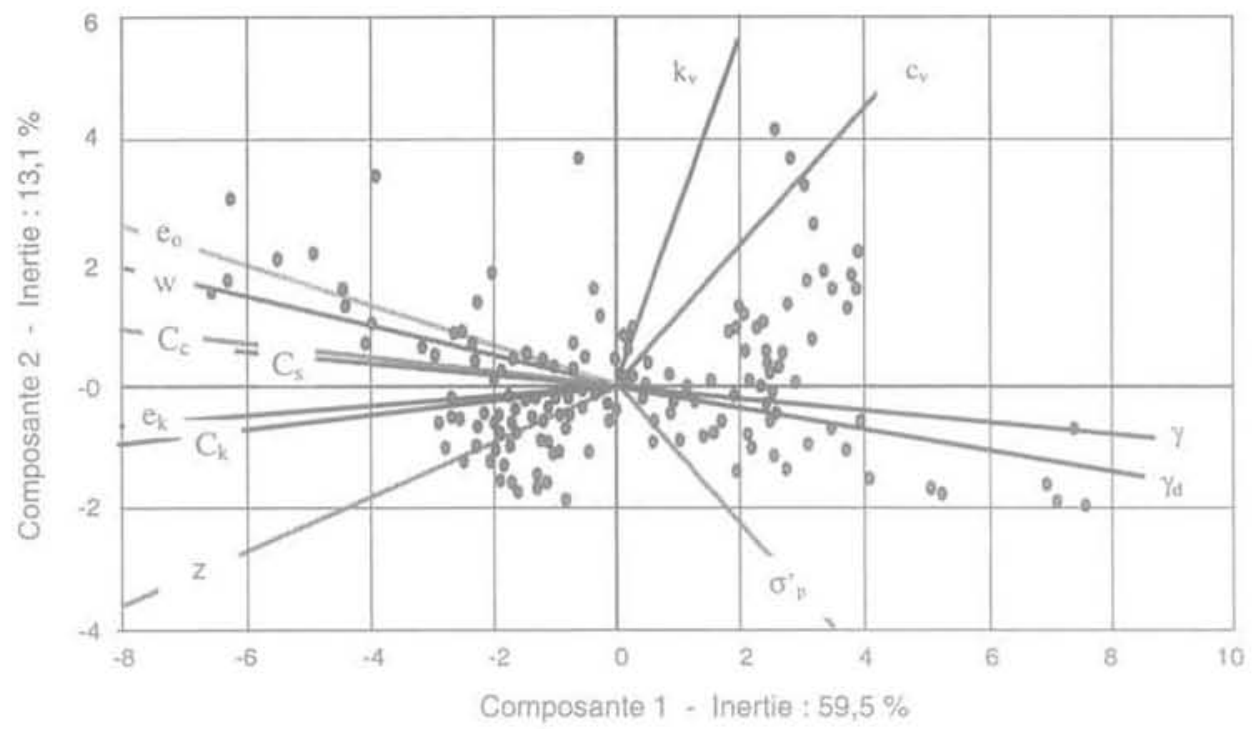

Plan factoriel 1-2 de l'analyse en composantes principales (ensemble des données).

Plot of factors 1 and 2 from factor anaiysis (all data).

Le rapprochement des vecteurs ou leur opposition montrent des relations marquées entre les paramètres, correspondant à des corrélations positives dans le cas de deux vecteurs proches l'un de l'autre et à des corrélations négatives en cas de vecteurs opposés.

Dans les quatre groupes ainsi définis, on retrouve des paramètres présentant des relations bien connues, comme par exemple, pour les vecteurs proches, $\gamma$ et $\gamma_{\mathrm{d}^{\prime}}$ avec la relation :

$$
\gamma=\gamma_{d}(1+w)
$$

qui fait intervenir $w$, dont le vecteur représentatif est pratiquement opposé à celui de $\gamma_{\mathrm{d}}$.

Pour d'autres paramètres, la relation parait simplement logique : ainsi, l'indice de compression C, matérialise la " capacité du sol à ètre comprimé », qui augmente avec l'indice des vides $e_{0}$. L'indice des vides varie comme la teneur en eau, et en sens inverse du poids volumique...
Les informations tirées de l'analyse en composantes principales doivent tenir compte des connaissances en mécanique des sols dans la manière de s'intéresser à certains paramètres. Ainsi, on peut observer que les variables w et e sont symétriques des variables $\gamma$ et $\gamma_{f}$ avec une représentativité de l'axe principal de $92,7 \%$ (Fig. 4). Ces résultats vont dans le sens des corrélations existant entre ces paramètres (tableau I).

L'interprétation de la position des vecteurs représentant les variables dans les plans factoriels permet donc de mettre en évidence des relations entre les caractéristiques géotechniques, qui découlent de la formulation des propriétés des sols ou de phénomènes liés à leur formation. Toutefois, pour des types de sols aussi bien étudiés que les argiles molles, cette analyse ne fait que retrouver des relations et corrélations bien connues en mécanique des sols.

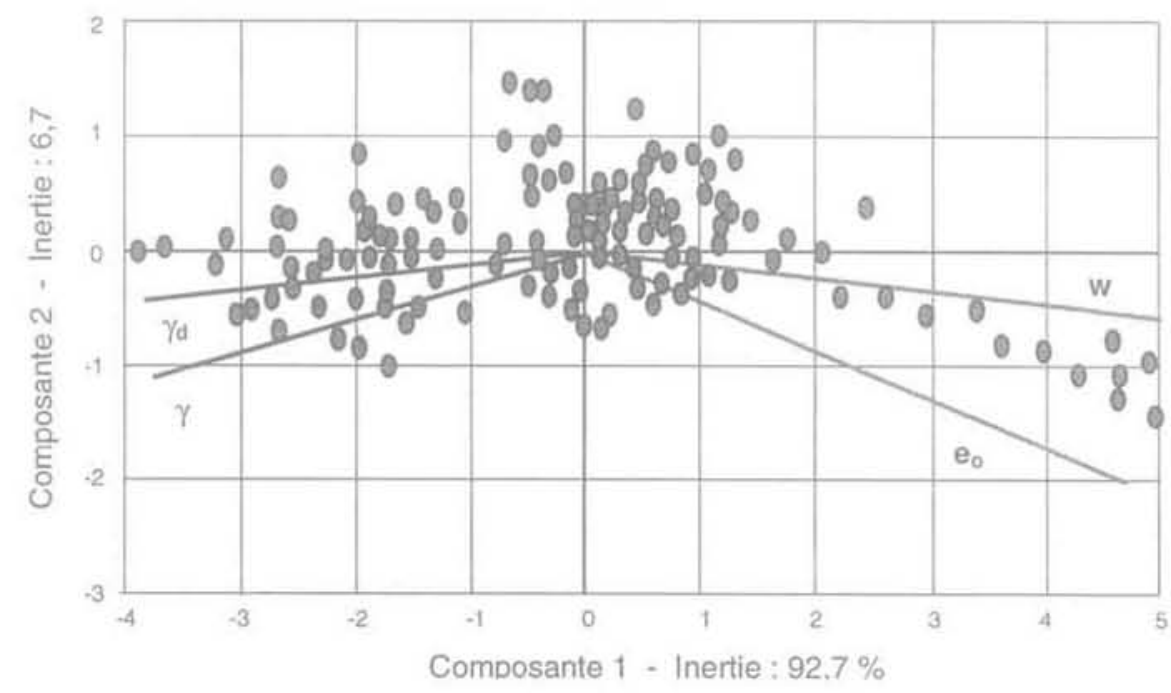

Plan factoriel 1-2 de l'analyse en composantes principales des paramètres $w, e_{\mathrm{o}^{\prime}} \gamma, \gamma_{\mathrm{d}}$. Plot of factors 1 and 2 from factor analysis $\left(w, e_{\alpha}, \gamma, \gamma_{d}\right)$. 
TABLEAU Coefficients de corrélation linéaire entre les paramètres $w$, $e_{0,} \gamma$ et $\gamma_{\text {d. }}$.

Correlation coefficients of soil parameters $w, e_{\text {. }}$ $\gamma$ et $\gamma_{\text {e: }}$

\begin{tabular}{c|c|c|c|c} 
& $w$ & $e_{0}$ & $\gamma$ & $\gamma_{d}$ \\
\hline$w$ & 1 & 0,95 & $-0,90$ & $-0,96$ \\
\hline$e_{0}$ & & 1 & $-0,76$ & $-0,86$ \\
\hline$\gamma$ & & & 1 & 0,97 \\
\hline$\gamma_{b}$ & & & & 1 \\
\hline
\end{tabular}

\section{Analyse au niveau des individus}

La représentation graphique de l'analyse en composantes principales peut être complétée par la numérotation des différents individus analysés. On peut ainsi définir des groupes représentant des individus proches les uns des autres. La détermination de ces groupes se fait "à la main » sur le graphique et la question de la séparation des zones reste généralement marcinale (quelques points des zones de transition peuvent prêter à ambiguîté). La figure 5 montre le plan factoriel de l'ensemble des données. La numérotation des individus permet de définir quatre groupes d'individus, qui correspondent à des couches superposées :

- couche 1 (point 1 à 7 ) : de 1,085 à $1,255 \mathrm{~m}$;

- couche 2 (points 15 à 25) : de 1,76 à 2,37 m ;

- couche 3 (points 33 à 84) : de 2,63 à 5,015 m;
- couche 4 (points 85 à 168) : de 5,28 à $9,075 \mathrm{~m}$.

On note le nombre relativement faible des points non comptabilisés dans la définition de ces couches: l'imprécision correspondante de la délimitation des couches est de l'ordre de $30 \mathrm{~cm}$.

L'analyse réalisée sur des plans factoriels limités à certains paramètres conduit à la définition de groupes d'amplitudes très proches de celles obtenues au niveau de l'ensemble des données. A titre d'exemple, le plan factoriel de l'analyse en composantes principales utilisant les paramètres $w, e, \gamma, \gamma$ (Fig. 6) montre une répartition des points différente de celle obtenue sur la figure 4 mais l'ordre et l'épaisseur des couches qui en résultent sont pratiquement identiques. On note que le plan factoriel obtenu dans ce cas possède une inertie de 99.4 $\%$, ce qui est dû au fait que, pour un sol saturé, il n'y a que deux paramètres indépendants parmi les quatre: en effet, il existe deux relations entre $w, e_{\alpha}, \gamma, \gamma_{d}$ :

$$
\begin{aligned}
& \gamma=\gamma_{d}(1+w) \\
& \mathrm{e}_{\mathrm{g}}=\frac{\gamma-\gamma_{\mathrm{d}}}{\gamma_{\mathrm{w}}+\gamma_{d}-\gamma}
\end{aligned}
$$

La figure 7 montre le plan factoriel 1-2 obtenu pour une autre combinaison de variables: $z, w, C$ et $\sigma^{\prime}$. Cette fois encore la disposition des points sur le graphique est différente, mais les couches obtenues sont les mêmes que dans les autres diagrammes.

Comme l'indique le tableau II, la prise en compte de tout ou partie des paramètres mesurés conduit à définir quatre couches de sol quasiment identiques à quelques centimètres près, c'est-à-dire à un ou deux niveaux de mesure près. Ces couches sont très proches de celles définies par la coupe géologique du sondage.

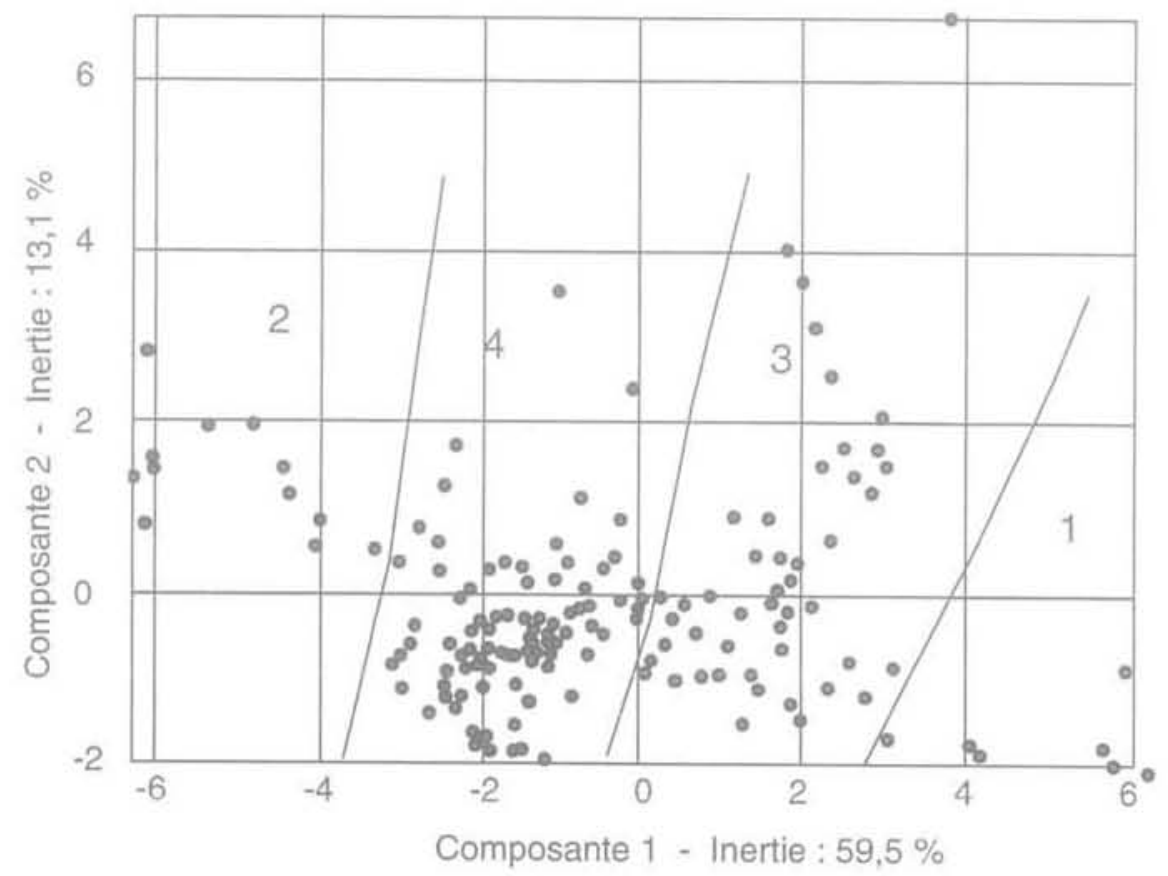

FIG.5 Plan factoriel 1-2 de l'analyse en composantes principales avec l'ensemble des paramètres, pour identification des individus.

Plot of factors 1 and 2 from factor analysis, including all soil parameters, for analysis at the level of individuals. 


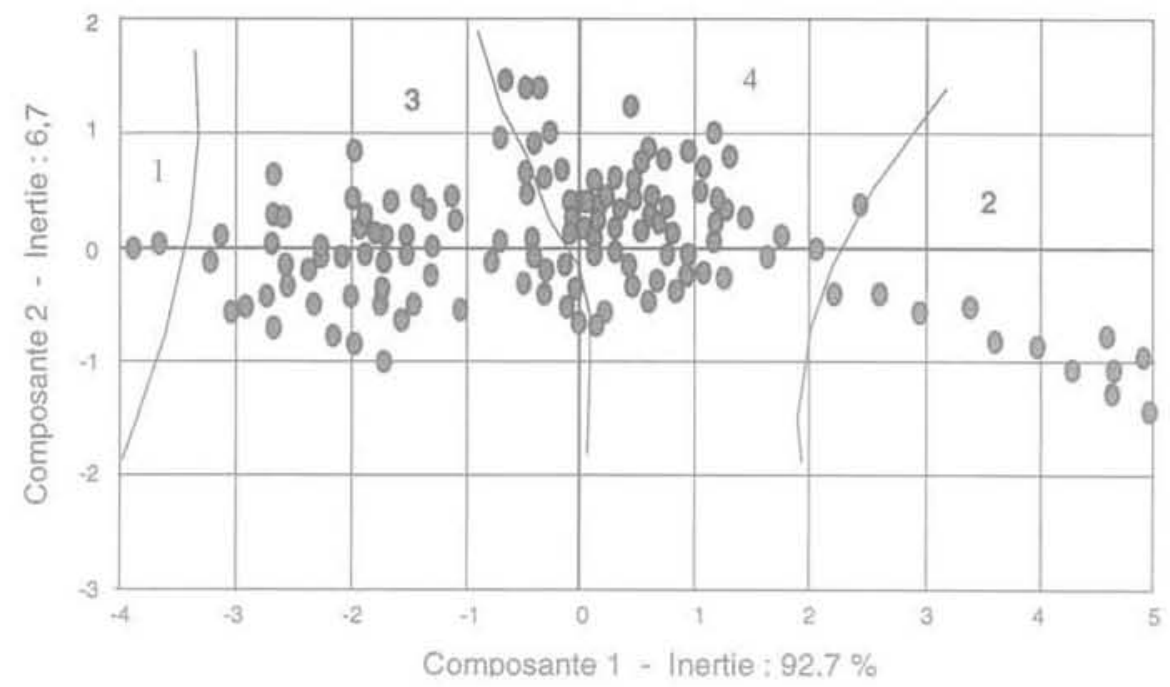

Fle. 6 Plan factoriel 1-2 de l'analyse en composantes principales avec les paramètres w, e., $\gamma$ et $\gamma_{d}$. Plot of factors 1 and 2 from factor analysis (soil parameters $w, e_{0,} \gamma$ and $\gamma_{\mathrm{d}}$ ).

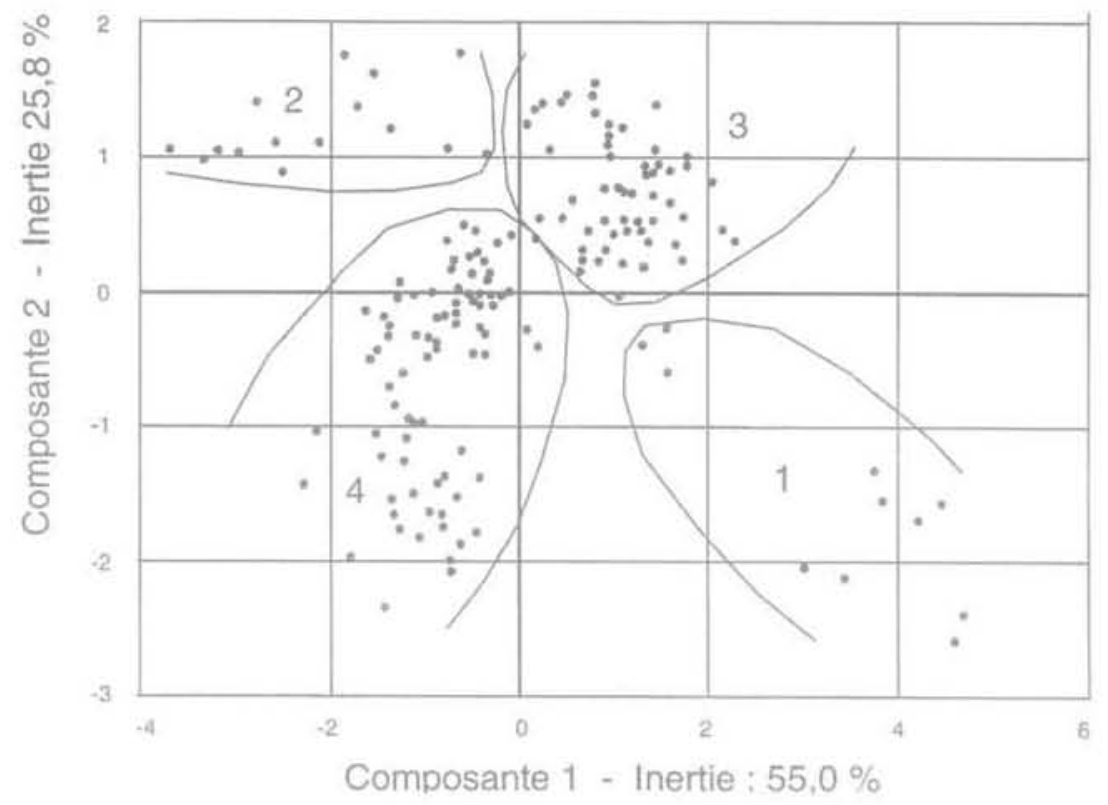

FG.7 Plan factoriel 1-2 de l'analyse en composantes principales avec les paramètres $z, w, C_{c}$ et $\sigma_{p}$. Plot of factors 1 and 2 from factor analysis (soil parameters $z, w, C_{\varepsilon}$ and $\sigma_{p}$ ).

On observe dans le tableau III que la représentativité des deux premiers axes factoriels est d'autant meilleure que le nombre de variables indépendantes (non liées par une relation mathématique) est faible. Les exceptions correspondent à des groupes de paramètres fortement corrélés (indice de compression et indice des vides, par exemple).

On remarque que, si l'analyse en composantes principales peut être utilisée pour déterminer les différentes couches de sol, elle ne permet pas de leur attribuer des caractéristiques géotechniques. Une étude statistique au niveau de chaque couche doit donc compléter les résultats. Cette étude statistique, menée sur l'ensemble des paramètres de chacune des couches mises en évidence, montre des coefficients de variation des paramètres par couche légèrement inférieurs ou en tout cas du mème ordre de grandeur que ceux mesurés sur l'ensemble des paramètres (une seule couche). A titre d'exemple, le tableau III donne les valeurs relatives au poids volumique $\gamma$ du sol. 
TABLEAUIII Zones déterminées par différentes analyses en composantes principales. Zones of similar solls (layers) obtained from various factor analyses.

\begin{tabular}{|c|c|c|c|c|c|c|c|c|c|c|c|c|c|c|c|c|c|}
\hline 2 & w & $\gamma$ & $\begin{array}{r}\text { Var } \\
\gamma_{0}\end{array}$ & $\begin{array}{l}\text { able } \\
\text { es }\end{array}$ & Intéc & ées & $\sigma_{p}$ & anal & $k$ & $e_{k}$ & $\mathrm{C}_{\mathrm{k}}$ & $\begin{array}{l}\text { Variables } \\
\text { non liées }\end{array}$ & $\begin{array}{c}\text { Inertie } \\
\text { du plan } \\
\text { factoriel } 1-2\end{array}$ & $\begin{array}{l}\text { Dé } \\
\text { Zone } 1 \\
\text { (m) }\end{array}$ & $\begin{array}{l}\text { finition d } \\
\text { Zone ? } \\
\text { (mi) }\end{array}$ & $\begin{array}{l}\text { les coucl } \\
\text { Zone } 3 \\
\text { (m) }\end{array}$ & $\begin{array}{l}\text { Zone } 4 \\
\text { (m) }\end{array}$ \\
\hline \multirow[t]{3}{*}{$\mathrm{x}$} & $x$ & $x$ & $\mathrm{x}$ & $\mathrm{x}$ & $x$ & $x$ & $x$ & $\mathrm{x}$ & $\mathrm{x}$ & $\mathrm{x}$ & $\mathrm{x}$ & 8 & $72,16 \%$ & $\begin{array}{l}1,085 \\
\text { à } 1,255\end{array}$ & $\begin{array}{l}1,76 \\
\text { à } 2,37\end{array}$ & $\begin{array}{l}2,63 \\
\text { à } 5,015\end{array}$ & $\begin{array}{l}5,28 \\
\text { à } 9,075\end{array}$ \\
\hline & $\mathrm{x}$ & $\mathrm{x}$ & $x$ & $\mathrm{x}$ & & & & & & & & 2 & $99,4 \%$ & $\begin{array}{l}1,085 \\
\text { à } 1,255\end{array}$ & $\begin{array}{l}1,76 \\
\text { à } 2,435\end{array}$ & $\begin{array}{l}2,63 \\
\text { à } 5,34\end{array}$ & $\begin{array}{l}5.40 \\
\text { à } 9,075\end{array}$ \\
\hline & $x$ & $\mathrm{x}$ & $\mathrm{x}$ & $x$ & $x$ & $\mathrm{x}$ & & & & & & 4 & $93.8 \%$ & $\begin{array}{l}1,085 \\
\text { à } 1.385\end{array}$ & $\begin{array}{l}1,76 \\
\text { aे } 2,435\end{array}$ & $\begin{array}{l}2,63 \\
\text { à } 5,015\end{array}$ & $\begin{array}{l}5,28 \\
\text { à } 9,075\end{array}$ \\
\hline $\mathrm{x}$ & $x$ & & & & & $\mathrm{x}$ & $\mathrm{x}$ & & & & & 4 & $80,8 \%$ & $\begin{array}{l}1,085 \\
\text { à } 1,385\end{array}$ & $\begin{array}{l}1,76 \\
\text { à } 2,435\end{array}$ & $\begin{array}{l}2,50 \\
\text { à } 5,015\end{array}$ & $\begin{array}{l}5,28 \\
\text { à } 9,075\end{array}$ \\
\hline \multirow[t]{3}{*}{$\mathrm{x}$} & & & & & & $x$ & $x$ & & & & & 3 & $82,2 \%$ & $\begin{array}{l}1,085 \\
\text { à } 1,385\end{array}$ & $\begin{array}{l}1.76 \\
\text { aे } 2,435\end{array}$ & $\begin{array}{l}2,50 \\
\text { à } 5,015\end{array}$ & $\begin{array}{l}5,28 \\
\text { à } 9,075\end{array}$ \\
\hline & $\mathrm{x}$ & & & & & $\mathrm{x}$ & $\mathrm{x}$ & & & & & 3 & $98.3 \%$ & $\begin{array}{l}1,085 \\
\text { à } 1,385\end{array}$ & $\begin{array}{l}1,76 \\
\text { à } 2,37\end{array}$ & $\begin{array}{l}2,435 \\
\text { à } 5,34\end{array}$ & $\begin{array}{l}5,40 \\
\text { aे } 9,075\end{array}$ \\
\hline & & & & & & $\mathrm{x}$ & $\mathrm{x}$ & $\mathrm{x}$ & $\mathrm{x}$ & $\mathrm{x}$ & $\mathrm{x}$ & 6 & $73,4 \%$ & $\begin{array}{l}1,085 \\
\text { à } 1,385\end{array}$ & $\begin{array}{l}1,76 \\
\text { à } 2,435\end{array}$ & $\begin{array}{l}2,50 \\
\text { à } 5,015\end{array}$ & $\begin{array}{l}5,28 \\
\text { aे } 9,075\end{array}$ \\
\hline$x$ & $\mathrm{x}$ & & & $\mathrm{x}$ & & & & $\mathrm{x}$ & $\mathrm{x}$ & $\mathrm{x}$ & $\mathrm{x}$ & 6 & $79,3 \%$ & $\begin{array}{l}1,085 \\
\text { aे } 1,255\end{array}$ & $\begin{array}{l}1,76 \\
\text { à } 2,37\end{array}$ & $\begin{array}{l}2.50 \\
\text { à } 5,28\end{array}$ & $\begin{array}{l}5,34 \\
\text { à } 9,075\end{array}$ \\
\hline
\end{tabular}

TABLEUIII Coefficients de variation du poids volumique par couche et global.

Coefficients of variation of unit weight for each layer and for the entire ground.

\begin{tabular}{l|c}
\hline Couche & Coefficient de variation \\
\hline 1 & $6,64 \%$ \\
\hline 2 & $2,82 \%$ \\
\hline 3 & $3,25 \%$ \\
\hline 4 & $2,25 \%$ \\
\hline Tout le sol & $5,65 \%$ \\
\hline
\end{tabular}

\section{5}

\section{Application au zonage géotechnique}

\section{1}

\section{Introduction}

Dans le cadre de l'étude géotechnique du site destiné à la construction d'un parking pour avions dans un aéroport, un grand nombre de sondages au pénétromètre statique a été réalisé dans une zone d'environ $1 \mathrm{~km}$ de longueur et $0,6 \mathrm{~km}$ de largeur (Fig. 8).

L'étude géotechnique a montré qu'il existe sur ce site une vallée fossile remplie de sédiments de faible résistance (sables et argiles) et dont la profondeur dépasse $20 \mathrm{~m}$. La position de la vallée a été déduite de l'analyse manuelle des courbes de variation de la résistance de cône $\mathrm{q}_{\mathrm{f}}$ en fonction de la profondeur. Les sols peu résistants ont été définis comme ayant une résis-

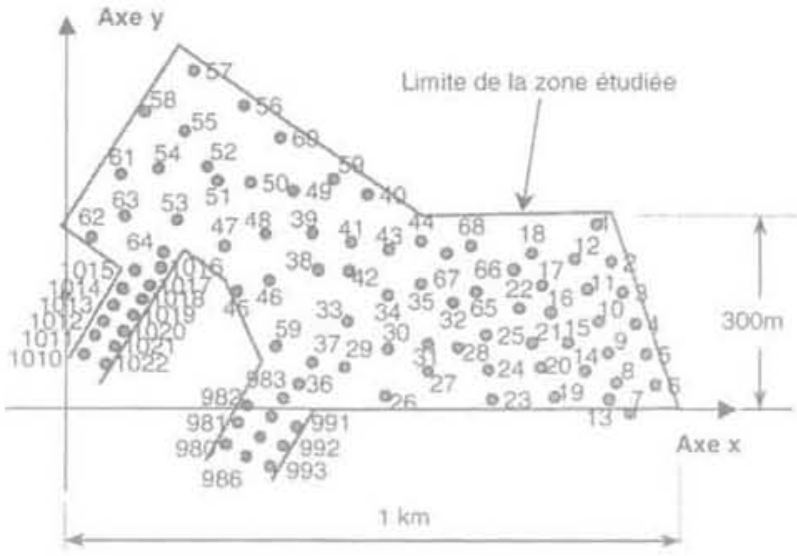

F6.8 Disposition des sondages pénétrométriques sur le site du projet. Layout of the penetration tests (CPT) at the construction site.

tance de cône inférieure à $2 \mathrm{MPa}$. La vallée est représentée sur la figure 9.

Les formations rencontrées à partir de la surface du sol sont successivement :

- de la terre végétale ou du remblai sur une épaisseur de 0,3 à 1,5 mètre ;

- de l'argile grise dont l'épaisseur peut atteindre 22 mètres. On peut rencontrer dans cette formation des argiles vasardes d'épaisseur variable ;

- de l'argile jaunâtre très consistante à dure, qui constitue le substratum pour le projet étudié.

La couche d'argile grise est absente au dehors de la vallée et a une épaisseur croissante quand on passe du bord au centre de la vallée. Des intercalations de sables et graviers sont rencontrées assez rarement jusqu'à une profondeur de 16 mètres; elles sont plus nombreuses entre 16 et 22 mètres de profondeur. Ces couches ont une épaisseur et une extension latérale très irrégulières. 


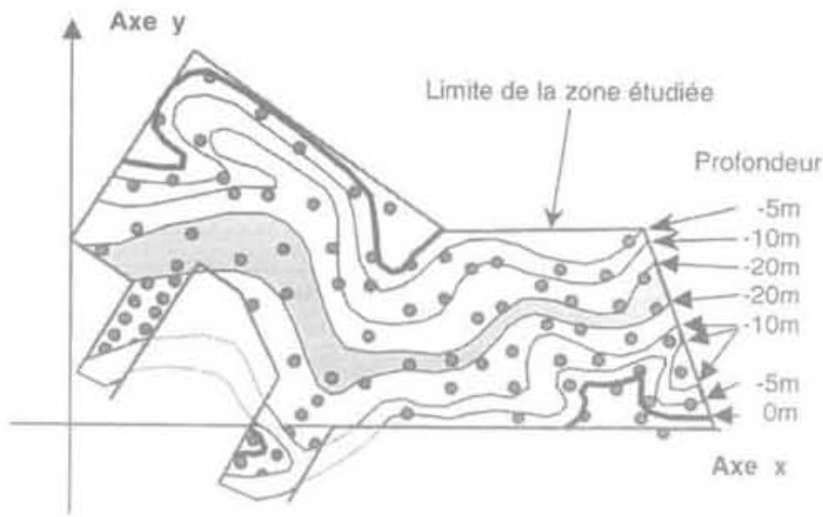

HG, 9 Position de la vallée fossile sur le site étudié.

Location of the hidden valley across the site.

La configuration de ce site a paru favorable pour essayer de délimiter l'étendue de la vallée fossile par une technique automatique d'analyse de données, en appliquant les outils de l'analyse factorielle.

A cette fin, comme cela avait été fait dans l'analyse manuelle, les pénétrogrammes (variations de la résistance de cône $q_{f}$ et du frottement latéral $f_{\mathrm{s}}$ en fonction de la profondeur) ont été simplifiés pour être enregistrés dans un tableau informatique. Les quatre-vingtdouze sondages ont été enregistrés à raison d'une valeur moyenne de $\mathrm{q}$ et $\mathrm{f}$ tous les $50 \mathrm{~cm}$. La figure 10 montre le pénétrogramme du sondage 25.

Le tableau de données comprend aussi cinq autres paramètres :

Résistance de cône $\mathrm{q}_{\mathrm{c}}$ (MPa) Frottement latéral unitaire $f_{\mathrm{s}} \times 10(\mathrm{MPa})$

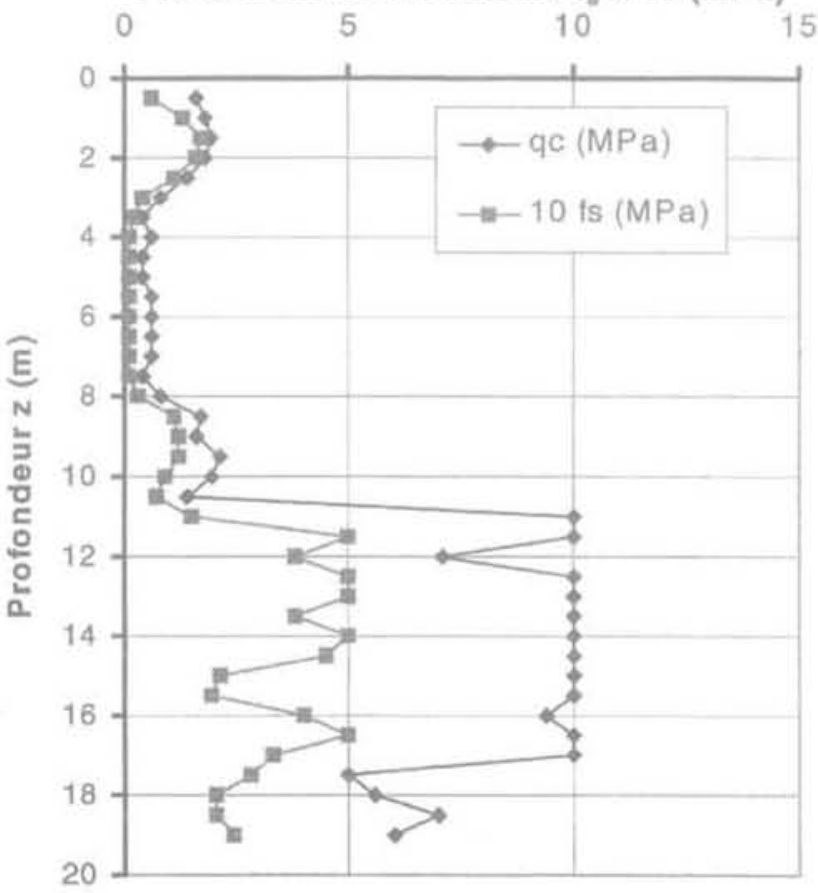

ก.. 10 Pénétrogramme du sondage 25. Penetrogramme of CPT souding Nr. 25.
- le numéro de l'enregistrement (les valeurs sont enregistrées dans l'ordre des profondeurs croissantes et par ordre croissant des numéros des sondages) ;

- le numéro du sondage :

- l'abscisse du point de sondage dans le repère choisi ; - l'ordonnée du point de sondage :

- la profondeur de la mesure.

\section{2}

\section{Étude dans la direction verticale}

Chaque sondage a d'abord été analysé séparément. Dans chacun des plans factoriels ainsi élaborés (où chaque individu apparaît par sa numérotation croissante avec la profondeur), on observe des zones oú ces individus sont proches les uns des autres : les familles ainsi constituées définissent des couches. Un exemple de résultat (sondage 25) est donné sur la figure 11, où l'on peut observer l'existence de quatre couches.

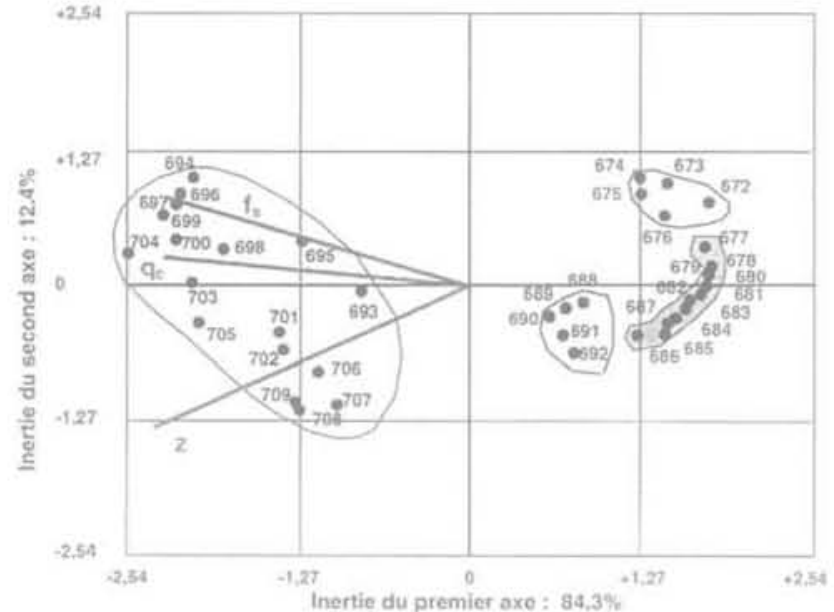

FG. 11 Biplot factoriel de l'analyse en composantes principales des données du sondage 25 (axes d'inertie 1 et 2 ). Mesures 672 à 709.

Plot of the first factors of data from CPT sounding $\mathrm{Nr}, 25$ (inertia axes 1 and 2) Data 672 to 709

Trois à sept (quatre à cinq le plus souvent) zones ou couches ont ainsi été déterminées par sondage. Chacune a fait l'objet d'une analyse statistique avec calcul de la moyenne, de la variance et du coefficient de variation des variables $q$ et $f$. On observe une très nette diminution du coefficient de variation par rapport au coefficient de variation mesuré sur toute la hauteur du sondage (tableau IV), ce qui confirme l'homogénéité des couches ainsi définies. On constate toutefois que ce phénomène est moins net dans les couches inférieures des sondages, où des intercalations de bancs de sable se font sentir. 
TABLEAUIV Quelques résultats statistiques obtenus sur les couches déduites de l'analyse en composantes principales. Some statistical properties of the CPT characteristics of each of the layers obtained from factor analysis.

\begin{tabular}{|c|c|c|c|c|c|c|c|c|c|}
\hline Sondage & Zone & $\begin{array}{l}\text { Numéros } \\
\text { des } \\
\text { individus }\end{array}$ & $\begin{array}{l}\text { Profondeur } \\
\text { z(m) }\end{array}$ & $\begin{array}{c}q_{c} \\
\text { moyenne } \\
\text { (MPa) }\end{array}$ & $\begin{array}{c}q^{2} \\
\left(\mathrm{MPa}^{2}\right)\end{array}$ & $\begin{array}{l}\text { q. } \\
\text { CV } \\
(\%)\end{array}$ & $\begin{array}{c}\text { is } \\
\text { moyenne } \\
(\mathrm{kPa})\end{array}$ & $\begin{array}{c}f_{5} \\
\sigma^{2} \\
(\mathrm{kPa})^{2}\end{array}$ & $\begin{array}{l}f \\
\mathrm{CV} \\
(\%)\end{array}$ \\
\hline \multirow[t]{5}{*}{30} & 1 & 809 à 811 & 1 à 2 & 2,53 & 0,173 & 16.4 & 120 & 100 & 8,3 \\
\hline & 2 & 812 à 823 & 2,5 a 8 & 1 & 0,075 & 27,3 & 37 & 300 & 49,8 \\
\hline & 3 & 824 à $B 41$ & 8,5 à 17 & 1.22 & 0.153 & 31,9 & 54 & 700 & 49,7 \\
\hline & 4 & 842 à 846 & 17,5 à 19,5 & 4,34 & 7,448 & 62,9 & 220 & 24800 & 71,6 \\
\hline & Global & 809 à 846 & & 1,88 & 3,855 & 104,6 & 86 & 10800 & 120,7 \\
\hline \multirow[t]{6}{*}{65} & 1 & 2013 à 2017 & 0,5 à 2,5 & 2,9 & 0,755 & 30 & 192 & 6600 & 42,4 \\
\hline & 2 & 2018 à 2025 & 3 à 6,5 & 0,93 & 0.054 & 25 & 34 & 100 & 22 \\
\hline & 3 & 2026 aे 2032 & 7 à 10 & 2,5 & 0,273 & 20,9 & 161 & 800 & 17,3 \\
\hline & 4 & 2033 à 2044 & 10,5 à 16 & 2,08 & 0,68 & 39,7 & 102 & 1600 & 39,8 \\
\hline & 5 & 2045 à 2052 & 16,5 à 20 & 7,35 & 7.003 & 36 & 346 & 8000 & 25,9 \\
\hline & Global & 2013 à 2052 & & 3,08 & 6,646 & 83,8 & 159 & 14300 & 75,3 \\
\hline
\end{tabular}

\section{3}

\section{Étude dans un plan horizontal}

La technique d'analyse en composantes principales a été utilisée pour analyser les résultats des essais pénétrométriques dans des plans horizontaux choisis à différentes profondeurs (le fichier de données a été conçu de manière à comporter l'altitude de chaque niveau de mesure), Pour les sondages dont la longueur est inférieure à 20 mètres, les données ont été complétées jusqu'à cette profondeur par une valeur correspondant à la dernière valeur mesurée (ce sont des sondages dans des argiles raides ne posant pas de problèmes géotechniques).

L'analyse a été réalisée sur la résistance de cône q, (qui est a priori le paramètre mesuré le plus représentatif pour les sols compressibles) et a consisté à établir des cartes de courbes isométriques de ce paramètre suivant la profondeur. L'intérêt de l'analyse en composantes principales est qu'elle permet des représentations factorielles où l'on peut analyser des ressemblances entre individus et les classer. Dans le cas présent, on analyse seulement deux variables $\mathrm{q}_{\mathrm{c}}$ et $\mathrm{f}_{\mathrm{s}}$ et toute la variabilité est représentée dans le plan factoriel 1-2. Le travail se décompose comme suit :

- à une profondeur donnée, traçage du plan factoriel calculé à l'aide des paramètres mesurés. Décomposition du plan en dix zones ou classes d'appartenance ; - sortie du même plan factoriel en faisant apparaitre cette fois le numéro de la mesure. Par comparaison avec la figure précédente, on sait alors à quelle classe appartient chaque sondage ;

- traçage, sur le plan de situation des sondages, des courbes correspondant aux limites de classes. Ce traçage s'effectue «à la main ») avec interpolation entre les points du graphe. Des formes de couches insolites ont parfois été trouvées par cette démarche (liées à l'imprécision du système, d'une part, et à la présence de niveaux de sables et graviers, d'autre part), mais, globalement, les indéterminations ont été très réduites et les cartes ainsi obtenues prêtent peu à discussions.

On dispose ainsi d'une carte pour chacune des profondeurs prises en compte, soit tous les 2,5 mètres. Les figures 12 et 13 montrent les courbes isométriques obtenues aux profondeurs de 10 et 20 mètres (la profondeur est donnée par rapport à l'altitude de référence, qui est celle de l'extrémité supérieure du sondage le plus élevé). L'analyse des courbes permet de préciser la situation et la forme de la vallée fossile ; elle donne une information sur la forme du fond de celle-ci (forme se rapprochant du $\mathrm{V}$ plutôt que du U).

La figure 14 compare la géométrie de la vallée à $20 \mathrm{~m}$ de profondeur (zone de résistances de cône $\mathrm{q}_{\text {. }}$ inférieures à $2 \mathrm{MPa}$ ) dans l'analyse qui vient d'être présentée et dans l'analyse manuelle effectuée lors de l'étude du site. On observe que la concordance est

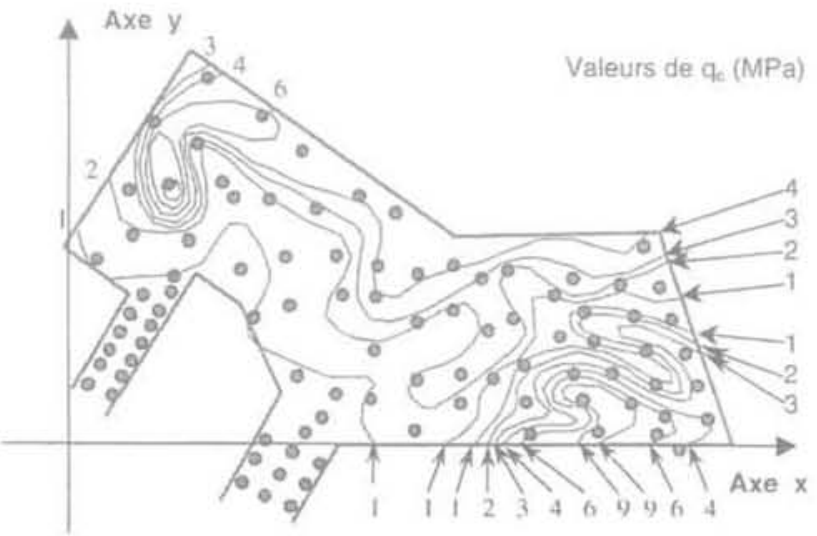

FIG. 12 Courbes isométriques de résistance de cône $\mathrm{q}_{\mathrm{c}}$ à la profondeur de $10 \mathrm{~m}$. Lines of equal cone resistance $\mathrm{q}_{\mathrm{c}}$ at $10 \mathrm{~m}$ depth. 


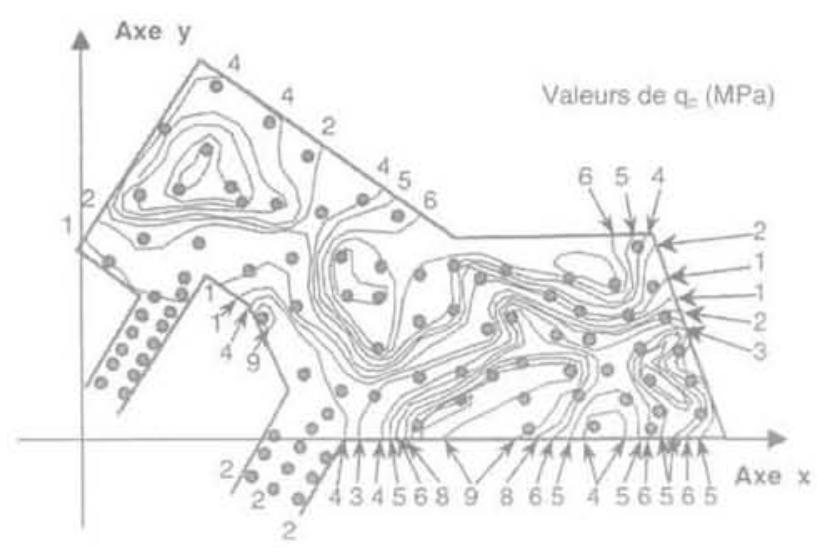

FGG.13 Courbes isométriques de résistance de cône à la profondeur de $20 \mathrm{~m}$. Lines of equal cone resistance $\mathrm{q}_{c}$ at $20 \mathrm{~m}$ depth.

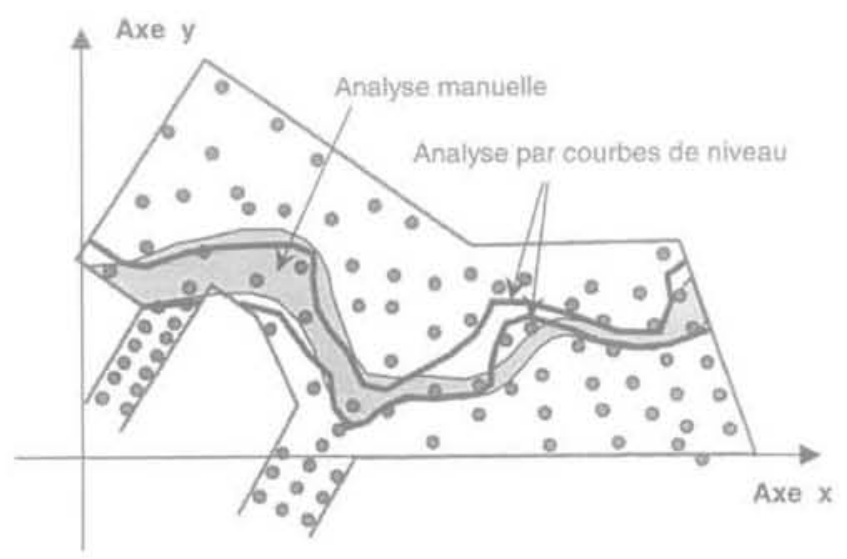

FIG. 14 Comparaison des formes de la vallée à $20 \mathrm{~m}$ de profondeur.

Comparison of the shapes of the valley at $20 \mathrm{~m}$ depth.

assez bonne. La principale différence est située au centre de la figure où la vallée est tracée de part et d'autre de deux sondages dans les deux analyses, ce qui est possible si l'on limite l'analyse à ce seul niveau.

La figure 15 compare les vallées à la profondeur de $10 \mathrm{~m}$. Cette fois encore on retrouve une allure compa-

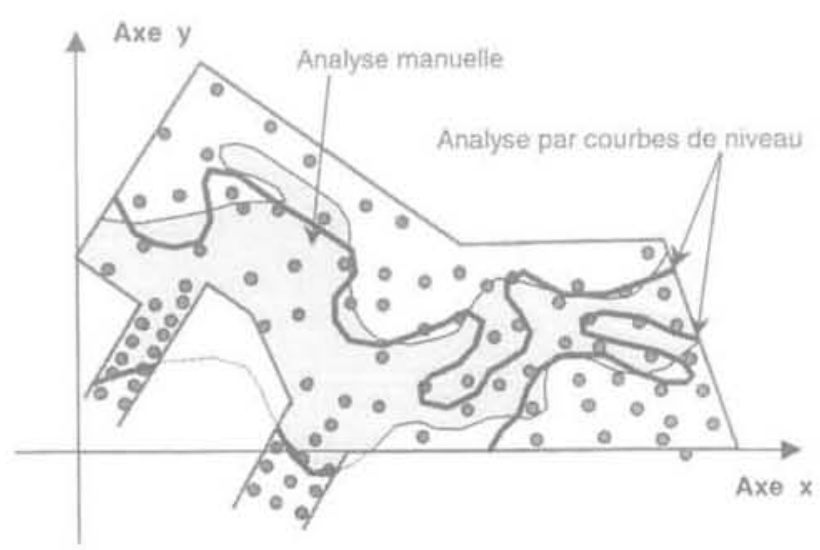

FG. 15 Comparaison des formes de la vallée à $10 \mathrm{~m}$ de profondeur.

Comparison of the shapes of the valley at $10 \mathrm{~m}$ depth. rable des zones de résistance de cône faibles $\left(\mathrm{q}_{\mathrm{c}}<\right.$ $2 \mathrm{MPa}$ ), avec des divergences d'appréciation sur quelques sondages.

Ces différences proviennent principalement des choix faits dans l'analyse manuelle globale, où la contrainte d'un rétrécissement progressif de la vallée en profondeur a conduit à éliminer des valeurs locale-

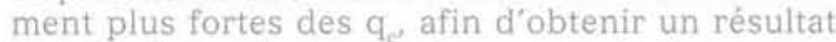
logique. Les analyses par niveau, quand elles ne sont pas corrigées par une analyse " tridimensionnelle » tenant compte des règles de la géomorphologie, peuvent en effet donner des résultats incohérents, comme on le voit dans ce cas sur la figure 16.

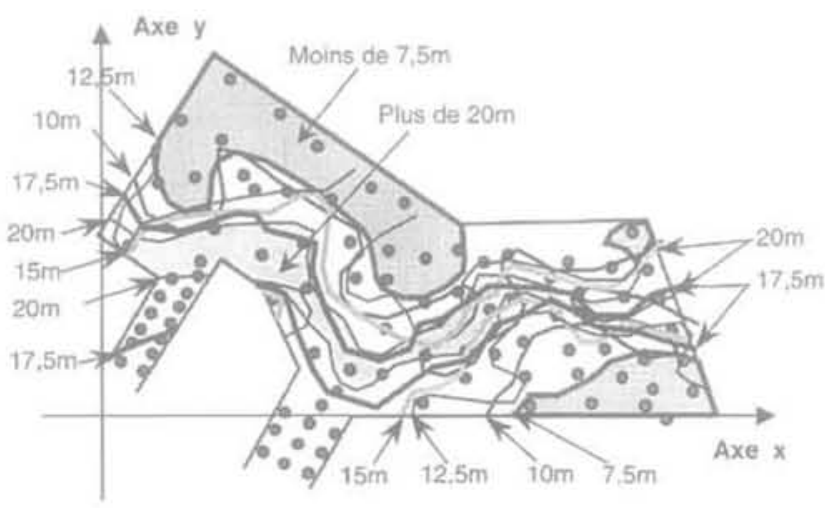

FIG.16 Comparaison des résultats des analyses par plans horizontaux.

Comparison of the results of the analyses made on horizontal planes.

\section{6}

\section{Conclusion}

Les deux applications de l'analyse factorielle (analyse en composantes principales) présentées dans cet article ont montré que cette technique d'analyse de données peut être appliquée avec succès à des données géotechniques. Pour l'étude des propriétés déduites d'une série importante d'essais cedométriques réalisés dans le même sondage, les relations connues pour lé comportement des argiles ont été retrouvées, mais sans qu'apparaissent des idées de relations nouvelles. Pour la recherche d'une ancienne vallée remblayée de sédiments plus compressibles dans une zone de sols plus raides, la démarche de l'analyse factorielle a permis de donner un caractère plus systématique à la recherche des frontières de cette vallée, mais elle doit être contrôlée pour assurer la cohérence du modèle du site et complètée pour déterminer les propriétés représentatives des différents sols identifiés. Le bilan de ces études exploratoires est donc satisfaisant pour la mise en œuvre et le bon fonctionnement du système d'analyse, qui utilise un logiciel spécialisé accessible à tous les utilisateurs. Mais les conclusions tirées des résultats bruts de l'analyse factorielle doivent être soigneusement pesées et confrontées aux connaissances disponibles pour éviter des interprétations erronées. 
Baghery S., Magnan J.-P. (1983) - "Analyse probabiliste de la stabilité et des tassements des remblais du site expérimental de Cubzac-les-Ponts ». Paris, Laboratoire central des ponts et chaussées, Rapport de recherche LPC, $n^{2} 122,71 \mathrm{p}$

Bertier P., Bouroche J.-M. (1977) - Analyse de données multidimensionnelfes Presses universitaires de France, $2^{\tau}$ éd. Boulefkhad T, (1986) - " influence de la variabilité des propriétés des sols sur le calcul des tassements des fondations superficielles $n$. Thèse de docteur ingénieur en génie civil présentée à l'univer. sité de Clermont II UFR de Recherche scientifique et technique.

Dagnelie P. (1977) - Analyse statistique à plusieurs variables. Presses agronomiques de Gembloux, diffusion VanderOyez réédition.

Favre J.-L. (1980) - « Milieu continu et milieu discontinu: mesure statistique inciirecte des paramètres rhéologiques et approche probabiliste de la sécurité ».
Thèse de doctorat d'Etat en sciences physiques, université Pierre-et-MarieCurie, Paris.

Haghgou M. (1983) - « Étude du compor. tement d'un remblal expérimental sur sol compressible n. Thèse de docteur ingénieur présentée à l'université Pierre-et-Marie-Curie, Paris VI.

Lebart L., Morineau A., Fenelon J.-P. (1982)Traitement des données statistiques. Méthodes et programmes, Paris, Dunod. $516 \mathrm{p}$. 